\title{
Review
}

\section{Impact of stress and hypertension on the cerebrovasculature}

\author{
Nada Elsaid ${ }^{1,2}$, Ahmed Saied ${ }^{1,2}$, Heba Kandil ${ }^{2,3}$, Ahmed Soliman ${ }^{2}$, Fatma Taher ${ }^{4}$, Mohiuddin Hadi ${ }^{5}$, \\ Guruprasad Giridharan ${ }^{2}$, Richard Jennings ${ }^{6}$, Manuel Casanova ${ }^{7}$, Robert Keynton ${ }^{8}$, Ayman El-Baz ${ }^{2, *}$ \\ ${ }^{1}$ Faculty of Medicine, Neurology Department, Mansoura University, 35516 Mansoura, Egypt, ${ }^{2}$ Bioimaging Laboratory, \\ Bioengineering Department, University of Louisville, Louisville, KY 40292, USA, ${ }^{3}$ Information Technology Department, \\ Faculty of Computer Science and Information, Mansoura University, 35516 Mansoura, Egypt, ${ }^{4}$ College of Technological \\ Innovation, Zayed University, 4783 Dubai, United Arab Emirates, ${ }^{5}$ Department of Radiology, University of Louisville, \\ Louisville, KY 40292, USA, ${ }^{6}$ Department of Psychiatry, University of Pittsburgh, Pittsburgh, PA 15260, USA, \\ ${ }^{7}$ Department of Biomedical Sciences, University of South Carolina, Greenville, SC 29208, USA, ${ }^{8}$ William States Lee \\ College of Engineering, University of North Carolina at Charlotte, Charlotte, NC 28223, USA
}

\section{TABLE OF CONTENTS}

1. Abstract

2. Introduction

3. Relation between stress and hypertension

3.1 Acute stress and hypertension

3.2 Chronic stress and hypertension

4. Cerebrovascular effects of stress

4.1 The effects of acute stress on the cerebral blood vasculature

4.2 The effects of chronic stress on the cerebral vasculature

5. The effects of hypertension on the cerebral vasculature

5.1 The structural effects of HTN on the cerebral vasculature

5.2 The functional effects of HTN on the cerebral vasculature

6. Quantification of the cerebral vasculature changes

6.1 Digital subtraction angiography (DSA)

6.2 Computed tomography (CT) and CT Angiography (CTA)

6.3 Magnetic resonance imaging (MRI), magnetic resonance angiography (MRA) and other similar techniques

6.4 Positron emission tomography (PET) and single photon emission computed tomography (SPECT)

6.5 Transracial doppler

6.6 Photoacoustic imaging (PAI)

6.7 Fast optical signals and event-related optical signal (EROS)

7. Risk stratification and clinical implication

8. Limitations and critical appraisal

9. Summary and conclusions

10. Author contributions

11. Ethics approval and consent to participate

12. Acknowledgment

13. Funding

14. Conflict of interest

15. References 


\section{Abstract}

Objectives: Both stress and hypertension (HTN) are considered major health problems that negatively impact the cerebral vasculature. In this article we summarize the possible relationship between stress and HTN. Methods: We conducted a systematic review of the literature using a database search of MEDLINE, PubMed, Scopus, and Web of Science. Results: Psychological stress is known to be an important risk factor for essential hypertension. Acute stress can induce transient elevations of blood pressure in the context of the fight-or-flight response. With increased intensity and duration of a perceived harmful event, the normal physiological response is altered, resulting in a failure to return to the resting levels. These changes are responsible for the development of HTN. Genetic and behavioral factors are also very important for the pathogenesis of hypertension under chronic stress situation. In addition, HTN and chronic stress may lead to impaired auto-regulation, regional vascular remodeling, and breakdown of the blood brain barrier (BBB). The effects of both HTN and chronic stress on the cerebral blood vessels shows that both have common structural and functional effects including endothelial damage with subsequent increased wall thickness, vessel resistance, stiffness, arterial atherosclerosis, and altered hemodynamics. Conclusion: Most of the above mentioned vascular effects of stress were primarily reported in animal models. Further in-vivo standardization of pathological vascular indices and imaging modalities is warranted. Radiological quantification of these cerebrovascular changes is therefore essential for in depth understanding of the healthy and diseased cerebral arteries functions, identification and stratification of patients at risk of cardiovascular and neurological adverse events, enactment of preventive measures prior to the onset of systemic HTN, and the initiation of personalized medical management.

\section{Introduction}

Stress is generally perceived to be an imbalance between environmental demands and a person's ability to meet them. Stress cannot be merely regarded as an environmental condition but as an interaction between a particular external exigency and an individual; thus, not every person will evaluate and react to a situation in the same way. According to the Medical Subject Headings (MeSH) stress is defined as a pathological maladaptive behavioral and hormonal response to extrinsic factors and abnormal conditions that affect its homeostasis. The daily events that activate the physiological responses can consequently cause to some extent, psychological wear and tear [1]. When emotional stressors are overwhelming, this condition is known as psychological stress. Modern lifestyles such as work-related and familial problems, social withdrawal, financial worries, and violence are some of the factors that can predispose or potentiate stress [2]. The burden of stress goes far beyond being a mental disorder with growing evidence of its potential role in the pathogenesis of various health problems like peptic ulcers, inflammatory/irritable bowel syndromes, metabolic syndrome, immune system dysfunction, cognitive dysfunction, and cardiovascular diseases including hypertension [2, 3]. This review will focus on the relation of stress to hypertension (HTN) and the possible sequelae for the cerebral vasculature.

Stress and hypertension (HTN) are correlated and constitute a major health risk. HTN creates risk for the nontransmittable cardiovascular diseases generally regarded as the major cause of death and disability worldwide. The number of hypertensive adults will reach 1.5 billion, approximately $30 \%$ of the world's population, by 2025 . The number of hypertension related mortalities was reported to be over 7 million in 2002; approximately $13 \%$ of all reported deaths [3]. The impact and the large magnitude of the associated morbidities as severe cardiovascular, renal and neurological complications are major concerns related to HTN [4]. Despite the many known risk factors for hypertension, including older age, family history, high body mass index, sedentary life, excess salt intake, fat, alcohol consumption, and smoking, the etiology of HTN remains not fully understood. Indeed, the genetic and behavioral factors known to be involved in HTN leave unexplained a substantial portion of the variability in outcomes [4].

Many factors suggest that psychological stress is an important risk factor for the development of essential hypertension. The objective of this manuscript is to review these possible links as well as the effect of both disorders on cerebral blood flow and vasculature.

\section{Relation between stress and hypertension}

\subsection{Acute stress and hypertension}

Acute stress can induce a transient elevation of blood pressure (BP), but it is still unclear whether this leads to chronic BP elevation. Sympathetic activation is held responsible for the transient elevation in BP observed during acute psychogenic stress. On exposure to an acute stressor, the fight-or-flight response is generated: increased heart rate, high cardiac output (COP), sodium and fluid retention, and visceral vasoconstriction resulting in circulatory redistribution favoring muscle and brain. This hyperreactivity is thought to be related to the central nervous system's perception of threat and initiation of copying with this threat resulting in activation of the limbic system with subsequent activation of the sympathetic nervous system and the sympathetic adrenal medullary system releasing catecholamines. In addition, a normal physiological response for stressful stimuli is activation of the hypothalamicpituitary-adrenal axis. This activation is characterized by the hypothalamic release of corticotropin-releasing factor 


\section{Acute stress}

Acute stressor

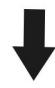

Fight / Flight defense response

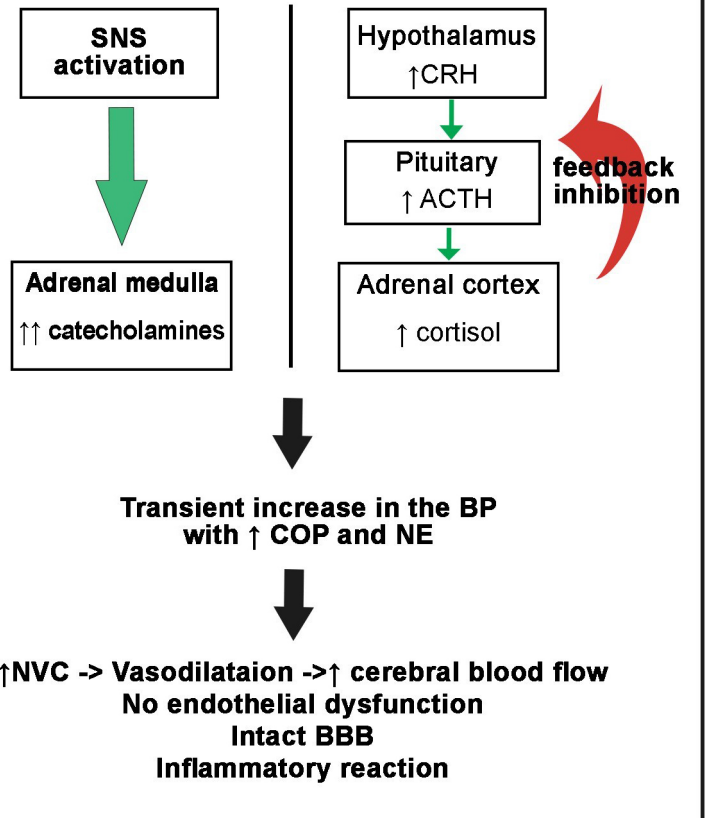

Chronic stress

Chronic overwelming stressor

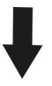

Defeat response

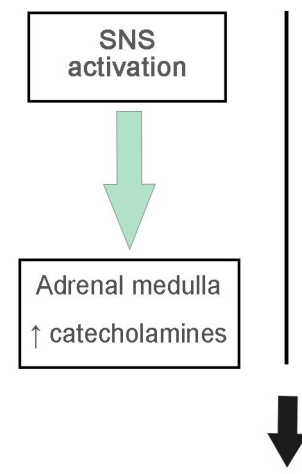

Chronic increase in the BP with normal COP and NE but with $\uparrow P R$

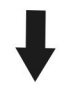

NVC dysfunction

Structural and functional endothelial dysfunction

BBB hyperpermeability

Marked inflammatory reaction

Fig. 1. Acute and chronic hormonal response to stress. SNS, sympathetic nervous system; CRH, corticotropin releasing hormone; ACTH, adrenocorticotropic hormone; BP, blood pressure; COP, cardiac output; NE, norepinephrine; NVC, neurovascular coupling; BBB, blood brain barrier; PR, peripheral resistance.

which in turn elicits pituitary release of adrenocorticotropic hormone, and cortisol. The production of corticotropinreleasing factor and adrenocorticotropic hormone is typically inhibited by negative feedback from increasing levels of catecholamines and cortisol with subsequent decrease of $\mathrm{BP}$ to its baseline levels [5]. The acutely elevated BP in response to stress is characterized by high cardiac output (CO) and norepinephrine levels. The CO tends to normalize first while the plasma norepinephrine level and sympathetic drive tend to last longer in the early phase of hypertension (Fig. 1).

\subsection{Chronic stress and hypertension}

The intensity and duration of stress are the most important factors in the determination of the risk for sustained BP elevations and hypertension. The pathophysiological mechanisms by which stress leads to sustained long term HTN are poorly understood. It may be that the recurrent activation of this system by similar stressors or some combination of the aforementioned factors, failure to return to the baseline levels after the stressful events, failure to accommodate to recurrent stressors is responsible for the development of hypertension [6]. Some authors suggest that the mechanism for development of HTN is attributed to a "defeat" response rather than a "defense" response.
The defeat reaction is described as loss of territorial control and failure to meet expectations in the event that chronic stress leads to a depressive mode. This state is mediated by the adrenocortical system in contrast to the defense response which is mediated by the sympathoadrenal system [7]. When exposed to chronic stressful stimulus, the normal physiological response is altered. The negative feedback mechanism is removed, and the central nervous system centers are reset to work at a slightly higher point. At first, the "desired" response is achieved by a potentiated autonomic cardiovascular drive. Later, with increased arteriolar responsiveness, a continuing hypertensive state is maintained with less sympathetic drive, with no need for either elevated cardiac output or for heightened norepinephrine release [8]. Contrary to the acutely elevated BP in response to stress, in the established phase of hypertension, both CO and norepinephrine levels are normal whereas the peripheral resistance becomes elevated. Although the neurogenic aspects of the early stages of hypertension can be easily linked to limbic activation, the association is still indeterminate when we consider the conversion from a high cardiac output to a high resistance state [8]. A potential role is likely for higher brain centers involved both in the perception and coping with stress and the organization of the neural and hormonal response, e.g., hippocampus, prefrontal cortex, 
and amygdala. Chronic stress exposure results in dysregulation of these structures which may lead to a disinhibition of the HPA and augmentation of the stress hormone release [8].

The stress induced physiological changes were the main focus of the investigations of the relationship between stress and HTN. The genetic predisposition plays a major role in determination of the level of personal cardiovascular reactivity with subsequent higher vulnerability to HTN and cardiovascular diseases in those with higher levels of cardiovascular reactivity in response to stress. The magnitude of the hemodynamic response is suggested to be genetically determined based on that it is least pronounced in normotensive controls without a familial history of hypertension, and maximal in individuals with labile hypertension with at least one hypertensive parent [9]. The speed by which the BP returns to its baseline levels appears to play a potential role in the development of HTN even more than the degree of reactivity. Thus, the physiological responses that primarily aim to maintain equilibrium can become harmful with persistent non necessary activation. Negative cognitive coping patterns such as rumination; have been linked to delayed BP recovery [9].

Generally, the intensity and the duration of the stressor are better determinants than the type of stressor in the development of the HTN. Chronic stress has been suggested to play a causative role in HTN based on its negative effects on the cardiovascular system including increased $\mathrm{BP}, \mathrm{COP}$, heart rate, left ventricular mass, and delayed poststress BP recovery in response to variable stressors (e.g., occupational stress, social environment stressor, low socioeconomic standard (SES), race related discrimination) [4].

Behavioral factors are also very important for the pathogenesis of hypertension under chronic stress situation. Chronic psychological stress could be associated with distorted lifestyle and mental distress as well as long-lasting allostatic load, contributing to the maintenance of blood pressure elevation [10].

\section{Cerebrovascular effects of stress}

4.1 The effects of acute stress on the cerebral blood vasculature

Stress is known to have clear, primarily vasoconstrictive effects on the peripheral vasculature, but the brain vessels are independent to some extent. Generally, the brain is dependent on its blood supply as the main source of energy and nutrients as it naturally lacks the ability to store intrinsic energy. Based on the brain's metabolic demands, it dynamically regulates the cerebral blood flow via a cascade of events involving neurovascular units. The neurovascular unit consists of the cerebral microvessels, glial cells (astrocytes, microglia, and oligodendrocytes), and neurons. Maintenance of precision and harmony between the endothelium and the neurons is the basis of normal cerebral circulation regulation. Neuronal activation leads to astrocytes being activated which in turn generate calcium dependent potassium and the release of vasodilators at the local arteriolar smooth muscle leading to vasodilatation. The basal cerebral vessels diameter (vascular tone) is maintained by the basal sympathetic nervous system discharge which ensures that changes in BP do not lead to overstretching of the cerebral arterioles, or lead to blood-brain barrier injury [11].

In the setting of acute psychological stresses, the neurovascular coupling is locally potentiated to increase the blood volume and perfusion in areas of increased neuronal activity that is to meet the blood oxygen level demand [5]. This can be visualized using perfusion functional MRI as an increase in cerebral blood flow in the ventral right prefrontal cortex and left insula/putamen area during acute psychological stress. In some studies, worry induction was associated with regional greater cerebral blood flow (CBF) in the visual cortex, thalamus, caudate and medial frontal cortex [12]. Concurrently, acute stress has been reported to raise the circulatory levels of proinflammatory cytokines (interleukin-6 (IL-6) and tumor necrosis factor (TNF- $\alpha$ )) increasing both the vulnerability to inflammatory disease and platelet activation [13].

\subsection{The effects of chronic stress on the cerebral vasculature}

Chronic stress, particularly in animal models, has been related to altered cerebral vasculature. Stress may impair the neurovascular balance responsible for matching the CBF to the brain metabolic demand every moment. This results in subtle brain alterations, eventually leading to chronic brain injury. Chronic stress is reported to affect the neurovascular coupling at the cellular level (neuron, astrocytes and more recently at the level of the endothelial cells [14]. In this review, we will focus on the chronic effect of stress that may translate into modification of the cerebral blood vessels.

\subsubsection{The structural effects of chronic stress on the cerebral vasculature}

In contrast to the defensive and adaptive nature of the acute cerebral vascular responses to stress, chronic stress induces a sustained hyperactivity of the hypothalamic pituitary axis (HPA) often referred to as "allostatic overload” (Fig. 1). Overstimulation of the autonomic nervous system (ANS) and HPA will eventually result in endothelial injury via a variety of downstream mechanisms. Hyperstimulation of the renin-angiotensin system leads to increased levels of homocysteine which induces endothelial damage. Increased cortisol levels directly decrease nitric oxide (NO; a powerful vasodilator) levels through the inhibition of endothelial NO synthase, and indirectly by reducing the production of reactive oxygen species (ROS) and oxidative stress. Also, chronic stress decreases the adenyl cyclase enzyme activity resulting in decrease cyclic adeno- 
sine monophosphate (cAMP). The persistently increased corticotropin-releasing factor results in a marked increase in the proinflammatory mediators and cytokines (Endothelin 1 , TNF- $\alpha$, Prostaglandin I2, IL-1 $\beta$, IL-6). All of these factors, in combination, result in cerebral endothelial injury and dysfunction [5]. Thus, chronic stress may contribute to intima-media thickening, the development of arterial stiffness and atherosclerosis of the cerebral blood vessels in ways similar to what has been previously reported in peripheral blood vessels [15].

Vascular angiogenesis dysfunction is another consequence of the stress induced endothelial injury. Neovascularization is an intrinsic physiologic tissue response to different stimuli through arteriogenesis (collateral remodeling) and angiogenesis (formation of new vessels from the existing vasculature). Brooks et al. [16] demonstrated a significant reduction in the density of cerebral microvessels in male lean rats after 8 weeks of unpredictable chronic mild stress (UCMS); however, Pearson-Leary and colleagues found that microvessel density in the brain was higher to provide metabolic support following increased neural activity. These opposing results may be due to the use of different stress models (social defeat paradigm vs. UCMS), with variable duration (7 days vs. 8 weeks), and the different animal models utilized (Lean Zucker rat vs. Sprague Dawley rat) [17]. The process of angiogenesis is normally maintained within the normal limits under the influence of 2 counteracting factors: vascular endothelial growth factors (VEGF) promoting angiogenesis, and thrombospondin-1 (TSP-1) promoting capillary regression and rarefaction. Brooks et al. [16] reported increased TSP-1 expression with either VEGF protein expression being unchanged or slightly elevated in obese Zucker rats. It was suggested that this effect is mediated by stress induced oxidative stress and free oxygen radicals [17].

The vascular endothelium also plays an important role in BBB integrity. The stress induced glucocorticoids released during HPA dysfunction affects the BBB directly, or through mast cell activation leading to increased BBB permeability evidenced by the extravasation of intravenous 99-Technetium glucoheptonate $\left({ }^{99} \mathrm{Tc}\right)$ into the brain parenchyma [18].

4.2.2 The functional effects of chronic stress on the cerebral vasculature

Chronic stress affects cerebrovascular functional reactivity [5]. The UCMS model has been used by a majority of researchers to induce depression-like behaviors in rodents by exposing them to mild daily randomized stressors. After 8 weeks of UCMS, significant pathological alterations were observed in the proximal large cerebral blood vessels (which represents up to $40 \%$ of the total cerebrovascular resistance and their functional response is critical in preventing pressure fluctuations from reaching the distal regions of the cerebral circulation). This was shown by a decrease in the middle cerebral artery (MCA) vasodilation in response to acetylcholine (a potent endothelialdependent dilator) and an increased MCA constriction response to serotonin (a potent cerebrovascular constrictor). These adaptations to chronic stress were coupled with increased levels of oxidative stress and reduced NO bioavailability in the cerebral vessels [19].

An immobilization model has been used by researchers to induce chronic stress while recording cerebral blood volume changes in the somatosensory cortex with an optical imaging system. Animals under stress by chronic restraint exhibited a decrease in their hemodynamic vascular response, with dilation of the small pial arterial in the somatosensory cortex during hind limb electrical stimulation, in association with downregulation of neuronal NO synthase and heme oxygenase-2 and enhanced inducible NO synthase (iNOS) expression. Parvalbumin expression in GABAergic interneurons was decreased as well as glutamate receptor- 1 in neurons, whereas the microglial activation was increased. [20]

Other researchers have focused on the effect of stress on the neurovascular coupling using a 7-days heterotypical stress paradigm in rats. The coupling effect was represented in amygdala brain slices after neuronal stimulation by measuring the dilation of the parenchymal arteriole (PA). After stress, significant reduction in the PAs to neuronal stimulation was shown, as well as the dilation of isolated PAs to external potassium released from astrocytic end feet during NVC, suggesting a dysfunction of the smooth muscle inwardly rectifying potassium (KIR) channels. It was postulated that the chronic stress resulted in a glucocorticoid-mediated decrease in functional potassium channels in the parenchymal arterioles myocytes, which rendered the arterioles less reactive to potassium ions released from astrocytic end feet during neurovascular coupling, leading to impairment of this process.

As the integrity of NVC is crucial for the neurons survival, its stress induced dysfunction may contribute to the pathophysiology of brain disorders [21].

Chronic social defeat stress was shown to be associated with enrichment in pathways that delineate the vascular response to injury. These pathways followed a temporal sequence of immune responses manifested in microglial activity, inflammation, oxidative stress, growth factor signaling, and wound healing (i.e., platelet aggregation, hemostasis, fibrinogen deposition, and angiogenesis) resulting in cerebrovascular microbleeds in scattered locations, vascular pathology and blood-brain barrier breakdown visualized by plasma immunoglobulins and erythrocytes within the parenchyma and perivascular spaces of the mice brains $[22,23]$.

The primary downstream effects of chronic stress exposure on cerebrovascular dysfunction are presented in Fig. 2. 


\section{Chronic stress}

\section{ANS \& HPA}

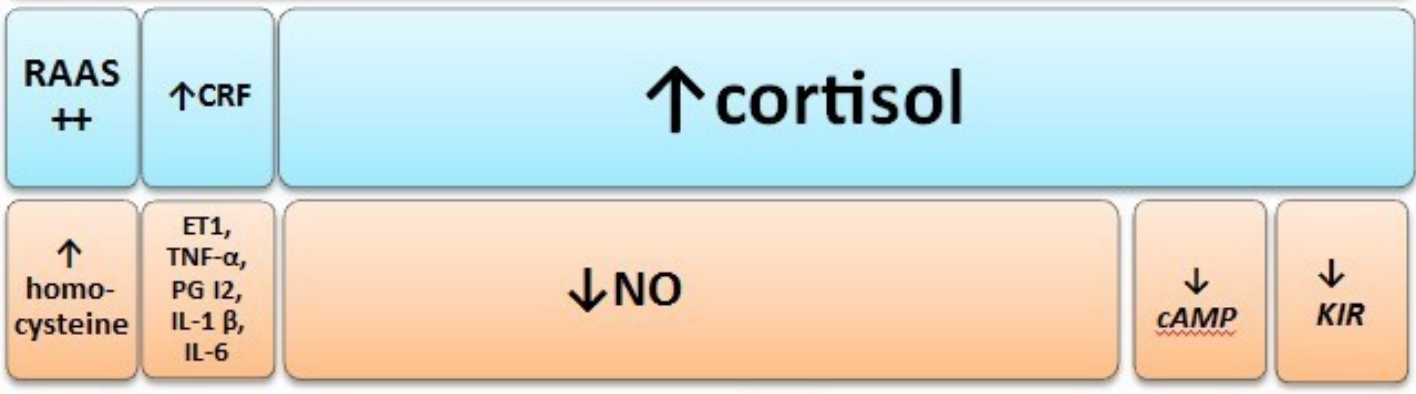

Endothelial injury

$\downarrow$ vasomotor reactivity

Increased wall thickness, vessel resistance, stiffness, and atherosclerosis, impaired auto-regulation, regional vascular remodeling, and BBB break down

Fig. 2. Schematic representation of the chronic stress induced cascade of events resulting eventually in cerebrovascular dysfunction.

\section{The effects of hypertension on the cerebral vasculature}

Hypertension also induces structural and functional changes in the cerebrovasculature either intrinsically or in combination with the effects of stress. It is hypothesized that the cerebrovascular changes and elevated cerebral arterial BP occur prior to the onset of systemic HTN and are thought to be the driver of systemic blood pressure changes [24].

5.1 The structural effects of HTN on the cerebral vasculature

The hallmark of HTN is an increase in peripheral resistance which is achieved by a reduction in lumen diameter and/or vessel number. Hypertension induces tangential stress on the arterial wall resulting in increased intralumenal pressure, arterial wall thickness, and wall-to-lumen ratio with a reduction in the lumen diameter, to maintain this stress within a physiological range. Several factors participate to the hypertrophy of cerebral arteries and arterioles, including: the trophic effects of the sympathetic perivascular innervation, the mechanical effects of the elevated intravascular pressure on the vascular wall through growth factors, oxidative stress, and reduced availability of NO. Hypertension also promotes the formation of atheroscle- rotic plaques in cerebral arteries and arterioles, and induces fibrinoid necrosis (lipohyalinosis) of penetrating arteries and arterioles [25].

Intracranial capillary regression and rarefaction has been reported for several models of hypertension induction; more so with the renal wrap, 2-kidney 2-clip, and deoxycorticosterone acetate (DOCA)-salt, than spontaneously hypertensive rats [26, 27]. Rarefaction of pial arteries was observed only by Sokolova et al. [26]. The arteriolar and capillary loss could result in chronic cerebral hypoperfusion through reduction of the cerebral blood flow [27]. The cerebral blood vessels structure is greatly affected by Hypertension. Structural and hemodynamic vessels dysfunction occur as a result of the reorganized cellular architecture and the vascular wall remodeling. Vascular occlusions may result from atherosclerosis and lipohyalinosis. The primary aim of the HTN induced vascular remodeling effects is to maintain the cerebral blood flow within the normal limits by increasing the vascular resistance in face of the elevated BP which is reported to be effective in the early stages of HTN. With time progression, this will result in regional blood flow reduction especially in the occipitotemporal, prefrontal cortex, and the hippocampus as reported in the older hypertensive patients. HTN also increases the BBB permeability and affects its capability of maintenance 
of the central nervous system homeostasis. Among potential mediators for the noted increased permeability are ROS, inflammation, and vascular remodeling [28].

5.2 The functional effects of HTN on the cerebral vasculature

Chronic HTN interferes with the local NVC mechanisms leading to impairment of the functional hyperemia with failure to meet up the increased metabolic demands of the regions of increased neuronal activity [28].

Cerebral arterial autoregulation is functioning when the mean arterial pressures range between 60 and $150 \mathrm{mmHg}$ to maintain the parenchymal perfusion at relatively constant levels. Outside of these values, the control of blood flow is lost and becomes dependent on mean arterial pressure. HTN increases the cerebral perfusion pressures needed for autoregulation of the CBF, i.e., shift of the pressure-flow curve to the right. Middle cerebral artery flow velocity monitoring by using transcranial doppler have shown that transient changes in BP still induce adaptive flow adjustments, indicating that the cerebral vessels are still able to compensate for the dynamic pressure changes despite the shift in the pressure-flow curve [29].

The increased sensitivity of the smooth muscles to calcium accounts for the increased myogenic tone and the shift in autoregulation. The myogenic reactivity is an innate response of the smooth muscle to stretch that is controlled by the endothelium through many mechanisms, such as NO, prostacyclin, and endothelium-derived hyperpolarizing factor (EDHF). The HTN induced endothelial dysfunction results in a reduction of these mediators thus promoting increased myogenic tone. Other suggested mediator of the increased myogenic tone is the 20-hydroxyeicosatetraenoic acid (20-HETE), which was found to be increased in the cerebral vessels of hypertensive rats [30].

\section{Quantification of the cerebral vasculature changes}

A review of the effects of both HTN and chronic stress on the cerebral blood vessels reveals that both have common effects. Indeed, both result in endothelial damage with subsequent increased wall thickness, vessel resistance, stiffness, and an atherosclerotic process that affects vessel diameter and tortuosity. These vascular changes lead to impaired autoregulation, regional vascular remodeling, and BBB break down. Quantification of these cerebrovascular changes is an essential step towards the better understanding of the healthy and diseased cerebral arteries functions. This new understanding may help to identify and stratify patients at risk of cerebral adverse events, enable preventive measures prior to the onset of systemic HTN, and optimize the medical management of those in need.

In this section, current and future trends in the imaging modalities will be discussed with a greater focus on technologies used for imaging blood vessels as a way of evaluating vascular function rather than parenchymal assessment.

\subsection{Digital subtraction angiography (DSA)}

DSA remains the gold standard methodology in the quantification and visualization of the cerebrovascular changes. However, the invasive nature of this technology and the attendant high cost limits its use. This neuroimaging modality and its post imaging processing software can give an idea about vessel diameter, tortuosity and cerebral hemodynamics [31]. Also, the cerebrovascular BP can be measured by a specialized sensor at the tip of the guiding catheter and micro catheter [32]. Intravascular ultrasound or the more recent optical coherence tomography (OCT) are ideal methods that provide a histopathological view of the vessel wall and measure the intimal and medial thickness [33].

\subsection{Computed tomography (CT) and CT Angiography} (CTA)

Conventional CT provides minimum information about the vascular system. Its sensitivity may be increased by contrast enhancement. CTA can be used to assess arterial sizes, evaluate blood flow to diagnose vascular conditions such as stenosis (narrowing of the blood vessel), occlusion, and atherosclerosis. Color-coded CTA allows better understanding of the cerebral hemodynamics, helping in the differentiation between anterograde and retrograde flow [34]. Micro-scale computed tomography (microCT) and nano-computed tomography (nanoCT) provides a highresolution volumetric representation of vascular structures and cerebral blood volume (CBV) measurements. CBV and $\mathrm{CBF}$ can be measured using perfusion $\mathrm{CT}$ allowing rapid qualitative and quantitative assessment of cerebral vascular hemodynamics [35].

6.3 Magnetic resonance imaging (MRI), magnetic resonance angiography (MRA) and other similar techniques

Magnetic resonance imaging (MRI) has a major role in the investigation of cerebrovascular diseases. Compared to CT and DSA, its advantages in diagnosing cerebrovascular pathology include its superior tissue contrast, its ability to visualize blood vessels without the use of a contrast agent, and its use of magnetic fields and radiofrequency pulses instead of ionizing radiation. In recent years, ultrahigh field MRI at 7Tesla (7T) has shown promise in the diagnosis of many cerebrovascular diseases. Parenchymal enhancement in the dynamic contrast-enhanced MRI (DCE-MRI) denotes BBB hyper permeability [36]. The role of Diffusion (DWI) and perfusion (PWI) weighted MRI in the early detection and assessment of stroke is increasing. The advantages of Susceptibility-Weighted Imaging (SWI) arise from its ability to detecting cerebral microbleeds, differentiate between arteries and veins, and between calcifi- 
cation and hemorrhage [37]. Recently available Intracranial Vessel Wall Imaging (IVW) modalities are used for direct assessment of the vessel wall; therefore, providing an upcoming field of interest to assess intracranial vascular abnormalities stemming from different pathologies [38]. Magnetic Resonance Angiography (MRA) has been used to visualize intracranial circulation and extracranial carotid vessels. MRA can be performed with or without contrast enhancement. A novel MRA-based framework and segmentation approach was developed to delineate cerebral blood vessels from the surrounding tissue for the detection of changes in cerebrovascular blood vasculature [39]. Functional MRI (fMRI) is another modality that helps in measuring changes in the regional blood flow to identify active areas in the brain [24]. Four-dimensional (4D) flow MRI; 2D phase contrast MRI (PC-MRI) and magnetic resonance black-blood thrombus imaging technique (MRBTI) are emerging modalities for cerebrovascular hemodynamics and CBF assessment [40].

6.4 Positron emission tomography (PET) and single photon emission computed tomography (SPECT)

Recently, multimodality PET has been used to evaluate the ischemic stroke related hypoxia and inflammation. SPECT was used as a guide for the use of antihypertensive medication in the immediate post- acute ischemic stroke period [41].

\subsection{Transracial doppler}

Transracial doppler (TCD) or color-coded duplex (TCCD) are noninvasive techniques that involve the use of 2-4 MHz probe to insonate the large intracranial vessels through specific acoustic windows and measure the CBF velocities and pulsatility indices [42].

\subsection{Photoacoustic imaging (PAI)}

Pulsed laser is used to produce thermal excitation and expansion of the tissue with subsequent generation of ultrasonic waves, i.e., photoacoustic effect. PAI depends on this technology to provide noninvasive visualization of the cerebrovasculature [43].

6.7 Fast optical signals and event-related optical signal (EROS)

Fast optical signals are recently developed imaging methods whose purpose is to provide spatio-temporal maps of the transmission of near-infrared (NIR) light through the intact human brain. The recording of fast optical signals can be time-locked to particular events (such as stimuli or responses), hence the name event-related optical signals (EROS). EROS is a new method for the noninvasive measurement of brain function, this technique is based on measures of the optical properties of cortical brain tissue, which change while the tissue is active. These changes are likely to be due to changes in light scattering, and are very rapid and localized, being related to phe- nomena occurring within or around the neuronal membrane. EROS, therefore, yields images of cortical activity that combine spatial specificity (i.e., they can be related to patches of tissue less than a cubic centimeter in size) with temporal resolution (i.e., they depict the time course of the neural activity in the cortical areas under measurement). A limitation of this technique is its reduced penetration into the head (less than $3-5 \mathrm{~cm}$ ). EROS appears to be a suitable technique for studying the time course of activity in selected cortical areas, and for providing a bridge between hemodynamic and electrophysiological imaging methods [44]. Most of the above-mentioned vascular effects of stress were primarily reported in animal models. Further in-vivo standardization of pathological vascular indices and imaging modalities is warranted. MRA may represent a promising, safe, convenient and cost-effective modality especially with the presence of novel segmentation frameworks which increases its sensitivity without the need of contrast enhancement.

\section{Risk stratification and clinical implication}

There is a need for risk stratification of hypertension in order to determine the type and intensity of treatment that is most appropriate. The current risk stratification is holistic and subjective, based on BP level, clinical risk factors, subclinical organ damage and established cardiovascular and renal diseases. The main risk factors include systolic and diastolic blood pressure (DBP) levels, age (males $>55$ years, females $>65$ years), smoking, hyperlipidemia, fasting plasma glucose of 5.6-6.9 mmol/L, impaired glucose tolerance test, central obesity and family history of premature cardiovascular disease (males $<55$ years, females $<65$ years) [45].

The detection of early cerebrovascular changes prior to the establishment of systemic HTN may provide an objective method for risk stratification which bears significant implications for clinical management. Based on detected changes, patients may gain access to early intervention and prophylaxis. Indeed, the combination of behavioral modification and neuroprotective medications may be promising in preventing HTN and subsequent complications such as vascular dementia and stroke.

\section{Limitations and critical appraisal}

The topic of stress is well studied and voluminous. In humans the focus is often attributed to the influential early work of Richard Lazarus [46]. Individual differences in general and specific differences in coping resources typically influence the reactions to stress. An example of such approach is the physiological and psychological distinction between reactions to an initial stressor as more consistent with threat or challenge. Human reactions are, at least, as variable to different stressors as the 
varieties noted in the review for animal studies. In animals repeated acute stress and sustained stress leads to the physiological changes though it is difficult to show any proof/specific mechanisms of this in humans. Longitudinal studies with the requisite physiological measurements are lacking. Mechanisms are more readily identified in the animal studies, but the generalization of both type of stressors and mechanism to humans problematic enough that it must be discussed. Causal directions remain in doubt. In humans, for example, the brain given its control over blood pressure has to be at least permissive when pressure rises to hypertensive levels. Correlational data relates blood pressure -at least in middle aged individuals- to brain function and cognitive output but it is not clear whether central changes preceded or followed peripheral effects of (the complex) pathophysiology of hypertension [47]. This emphasizes the importance of quantification of these cerebrovascular changes as an essential step towards the better understanding of the healthy and diseased cerebral arteries functions.

\section{Summary and conclusions}

Life stressors are almost experienced by everyone. However, the perception of stress varies from one to another and from a stressor to the other based on the duration and the intensity of the stressor. Chronic stress plays a major role in the pathogenesis of HTN especially in those with greater sensitivity of their neural pathways mediating the cardiovascular responses to stress. Exposure to chronic stress induces a cascade of events resulting eventually in cerebrovascular dysfunction with structural and functional effects similar to those induced by HTN as endothelial injury with increased wall thickness, vessel resistance, stiffness, arterial atherosclerosis, and impaired auto-regulation. Early radiological quantification of these cerebrovascular changes may predict the risk of cardiovascular and neurological adverse events even prior to the onset of systemic HTN helping in prevention and early treatment.

\section{Author contributions}

Conceptualization-NE, AS (Ahmed Saied), HK, AS (Ahmed Soliman), MH, RJ, AE-B; validation-NE, AS (Ahmed Saied), HK, MH, MC; writing — original draft preparation-NE, AS (Ahmed Saied); writing - review and editing-NE, AS (Ahmed Saied), HK, AS (Ahmed Soliman), FT, MH, GG, RJ, MC, RK, AE-B; supervisionGG, RK, AE-B; Financial support-FT; All authors have read and agreed to the published version of the manuscript.

\section{Ethics approval and consent to participate}

Not applicable.

\section{Acknowledgment}

Thanks to all the peer reviewers for their opinions and suggestions.

\section{Funding}

This research received no external funding.

\section{Conflict of interest}

The authors declare no conflict of interest.

\section{References}

[1] Kearney PM, Whelton M, Reynolds K, Muntner P, Whelton PK, He J. Global burden of hypertension: analysis of worldwide data. The Lancet. 2005; 365: 217-223.

[2] WHO. The world health report 2002—Reducing Risks, Promoting Healthy Life. Available at: https://www.who.int/whr/2002/ en/ (Accessed: 27 May 2019).

[3] Spruill TM. Chronic psychosocial stress and hypertension. Current Hypertension Reports. 2010; 12: 10-16.

[4] McEwen BS. Physiology and neurobiology of stress and adaptation: central role of the brain. Physiological Reviews. 2007; 87: 873-904.

[5] Burrage E, Marshall KL, Santanam N, Chantler PD. Cerebrovascular dysfunction with stress and depression. Brain Circulation. 2018; 4: 43-53.

[6] Hu B, Liu X, Yin S, Fan H, Feng F, Yuan J. Effects of psychological stress on hypertension in middle-aged Chinese: a crosssectional study. PLoS ONE. 2015; 10: e0129163.

[7] Henry JP, Stephens PM, Ely DL. Psychosocial hypertension and the defense and defeat reactions. Journal of Hypertension. 1986; 4: 687-697.

[8] Julius S. Transition from high cardiac output to elevated vascular resistance in hypertension. American Heart Journal. 1988; 116: 600-606.

[9] Brosschot JF, Pieper S, Thayer JF. Expanding stress theory: prolonged activation and perseverative cognition. Psychoneuroendocrinology. 2005; 30: 1043-1049.

[10] Straub SV, Bonev AD, Wilkerson MK, Nelson MT. Dynamic inositol trisphosphate-mediated calcium signals within astrocytic endfeet underlie vasodilation of cerebral arterioles. The Journal of General Physiology. 2006; 128: 659-669.

[11] Munakata M. Clinical significance of stress-related increase in blood pressure: current evidence in office and out-of-office settings. Hypertension Research. 2018; 41: 553-569.

[12] Karim HT, Tudorascu DL, Butters MA, Walker S, Aizenstein HJ, Andreescu C. In the grip of worry: cerebral blood flow changes during worry induction and reappraisal in late-life generalized anxiety disorder. Translational Psychiatry. 2017; 7: e1204.

[13] Marsland AL, Walsh C, Lockwood K, John-Henderson NA. The effects of acute psychological stress on circulating and stimulated inflammatory markers: a systematic review and metaanalysis. Brain, Behavior, and Immunity. 2017; 64: 208-219.

[14] McEwen BS, Nasca C, Gray JD. Stress Effects on Neuronal Structure: Hippocampus, Amygdala, and Prefrontal Cortex. Neuropsychopharmacology. 2016; 41: 3-23.

[15] von Känel R, Hepp U, Traber R, Kraemer B, Mica L, Keel M, et al. Measures of endothelial dysfunction in plasma of patients with posttraumatic stress disorder. Psychiatry Research. 2008; 158: 363-373.

[16] Brooks S, Branyan KW, DeVallance E, Skinner R, Lemaster K, Sheets JW, et al. Psychological stress-induced cerebrovascular 
dysfunction: the role of metabolic syndrome and exercise. Experimental Physiology. 2018; 103: 761-776.

[17] Pearson-Leary J, Eacret D, Chen R, Takano H, Nicholas B, Bhatnagar S. Inflammation and vascular remodeling in the ventral hippocampus contributes to vulnerability to stress. Translational Psychiatry. 2017; 7: e1160.

[18] Esposito P, Gheorghe D, Kandere K, Pang X, Connolly R, Jacobson $\mathrm{S}$, et al. Acute stress increases permeability of the bloodbrain-barrier through activation of brain mast cells. Brain Research. 2001; 888: 117-127.

[19] Mineur YS, Prasol DJ, Belzung C, Crusio WE. Agonistic behavior and unpredictable chronic mild stress in mice. Behavior Genetics. 2003; 33: 513-519.

[20] Lee S, Kang B, Shin M, Min J, Heo C, Lee Y, et al. Chronic Stress Decreases Cerebrovascular Responses during Rat Hindlimb Electrical Stimulation. Frontiers in Neuroscience. 2015; 9: 462.

[21] Longden TA, Dabertrand F, Hill-Eubanks DC, Hammack SE, Nelson MT. Stress-induced glucocorticoid signaling remodels neurovascular coupling through impairment of cerebrovascular inwardly rectifying $\mathrm{K}+$ channel function. Proceedings of the $\mathrm{Na}$ tional Academy of Sciences of the United States of America. 2014; 111: 7462-7467.

[22] Lehmann ML, Weigel TK, Cooper HA, Elkahloun AG, Kigar SL, Herkenham M. Decoding microglia responses to psychosocial stress reveals blood-brain barrier breakdown that may drive stress susceptibility. Scientific Reports. 2018; 8: 11240.

[23] Lehmann ML, Poffenberger CN, Elkahloun AG, Herkenham M. Analysis of cerebrovascular dysfunction caused by chronic social defeat in mice. Brain, Behavior, and Immunity. 2020; 88: 735-747.

[24] Jennings JR, Muldoon MF, Ryan C, Gach HM, Heim A, Sheu LK, et al. Prehypertensive Blood Pressures and Regional Cerebral Blood Flow Independently Relate to Cognitive Performance in Midlife. Journal of the American Heart Association. 2017; 6: e004856.

[25] Hayashi K, Naiki T. Adaptation and remodeling of vascular wall; biomechanical response to hypertension. Journal of the Mechanical Behavior of Biomedical Materials. 2009; 2: 3-19.

[26] Sokolova IA, Manukhina EB, Blinkov SM, Koshelev VB, Pinelis VG, Rodionov IM. Rarefication of the arterioles and capillary network in the brain of rats with different forms of hypertension. Microvascular Research. 1985; 30: 1-9.

[27] Paiardi S, Porteri E, Rodella L, De Ciuceis C, Boari GE, Rezzani $\mathrm{R}$, et al. Angiotensin Receptor Blockers and Ace Inhibitors Prevent Microvascular Rarefaction in the Skeletal Muscle of Spontaneously Hypertensive Rats. High Blood Pressure \& Cardiovascular Prevention. 2007; 14: 145-196.

[28] Jennings JR, Muldoon MF, Ryan C, Price JC, Greer P, SuttonTyrrell K, et al. Reduced cerebral blood flow response and compensation among patients with untreated hypertension. Neurology. 2005; 64: 1358-1365.

[29] Traon AP, Costes-Salon M, Galinier M, Fourcade J, Larrue V. Dynamics of cerebral blood flow autoregulation in hypertensive patients. Journal of the Neurological Sciences. 2002; 195: 139144.

[30] Pires PW, Dams Ramos CM, Matin N, Dorrance AM. The effects of hypertension on the cerebral circulation. American Journal of Physiology. Heart and Circulatory Physiology. 2013; 304: H1598-H1614.

[31] Elsaid N, Saied A, Joshi K, Nelson J, Baumgart J, Lopes D. 2D Parametric Parenchymal Blood Flow as a Predictor of the Hemorrhagic Events after Endovascular Treatment of Acute Ischemic Stroke: a Single-Center Retrospective Study. Interventional Neurology. 2018; 7: 522-532.

[32] Blanco PJ, Müller LO, Spence JD. Blood pressure gradients in cerebral arteries: a clue to pathogenesis of cerebral small vesse disease. Stroke and Vascular Neurology. 2017; 2: 108-117.

[33] Guerrero BP, Pacheco CD, Saied A, Joshi K, Rodríguez C, Martínez-Galdámez M, et al. First Human Evaluation of Endothelial Healing after a Pipeline Flex Embolization Device with Shield Technology Implanted in Posterior Circulation Using Optical Coherence Tomography. Neurointervention. 2018; 13: 129-132.

[34] Thierfelder KM, Havla L, Beyer SE, Ertl-Wagner B, Meinel FG, von Baumgarten L, et al. Color-coded cerebral computed tomographic angiography: implementation of a convolution-based algorithm and first clinical evaluation in patients with acute ischemic stroke. Investigative Radiology. 2015; 50: 361-365.

[35] Wintermark M, Sincic R, Sridhar D, Chien JD. Cerebral perfusion CT: technique and clinical applications. Journal of Neuroradiology. 2008; 35: 253-260.

[36] Harteveld AA, van der Kolk AG, Zwanenburg JJM, Luijten PR, Hendrikse J. 7-T MRI in Cerebrovascular Diseases: Challenges to Overcome and Initial Results. Topics in Magnetic Resonance Imaging. 2016; 25: 89-100.

[37] Kawada T. Re: Diagnostic performance of PWI/DWI MRI parameters in discriminating hyper-acute versus acute ischaemic stroke: finding the best thresholds. Clinical Radiology. 2012; 67: 1218; author reply 1218-1218; author reply 1219.

[38] Zhu X, Wang W, Liu Z. High-resolution Magnetic Resonance Vessel Wall Imaging for Intracranial Arterial Stenosis. Chinese Medical Journal. 2016; 129: 1363-1370.

[39] Kandil H, Soliman A, Taher F, Mahmoud A, Elmaghraby A, ElBaz A. 'Using 3-D CNNs and Local Blood Flow Information to Segment Cerebral Vasculature', Signal Processing and Information Technology (ISSPIT) 2018 IEEE International Symposium on. Louisville, USA. 2018.

[40] Turski P, Scarano A, Hartman E, Clark Z, Schubert T, Rivera L, et al. Neurovascular 4DFlow MRI (Phase Contrast MRA): emerging clinical applications. Neurovascular Imaging. 2016; 2: 8.

[41] Heiss W. PET imaging in ischemic cerebrovascular disease: current status and future directions. Neuroscience Bulletin. 2014 30: 713-732.

[42] Elsaid N, El-Mitwalli A, Farrag AM, Abdel-Salam M. Outcome and prognosis of middle cerebral artery occlusive disease in a sample of Egyptian patients: A prospective hospital-based study. African Journal of Neurological Sciences. 2020; 39: 113123.

[43] Tang J, Dai X, Jiang H. Wearable scanning photoacoustic brain imaging in behaving rats. Journal of Biophotonics. 2016; 9: 570-575.

[44] Gratton G, Fabiani M. The event-related optical signal: a new tool for studying brain function. International Journal of Psychophysiology. 2001; 42: 109-121.

[45] Kannel WB. Hypertension: Reflections on Risks and Prognostication. Medical Clinics of North America. 2009; 93: 541-558.

[46] Lazarus RS, Lazarus RS. Emotion and adaptation. Oxford University Press on Demand: Oxford. 1991.

[47] Hestad K, Engedal K, Schirmer H, Strand BH. The effect of blood pressure on cognitive performance. An 8-year followup of the Tromsø Study, comprising people aged 45-74 Years. Frontiers in Psychology. 2020; 11: 607.

Keywords: Stress; Hypertension; Cerebrovascular; Imaging

Send correspondence to: Ayman El-Baz, Bioimaging Laboratory, Bioengineering Department, University of Louisville, Louisville, KY 40292, USA, E-mail: aselba01@louisville.edu 